\title{
Diversité des espèces cultivées et contraintes à la production en agriculture maraîchère au Togo
}

\author{
Madjouma KANDA ${ }^{1 *}$, Sêmihinva AKPAVI ${ }^{1}$, Kpérkouma WALA ${ }^{1}$, \\ Gbandi Djaneye-BOUNDJOU ${ }^{2}$ et Koffi AKPAGANA ${ }^{1}$ \\ ${ }^{1}$ Laboratoire de Botanique et Ecologie Végétale, Faculté des Sciences, Université de Lomé, BP 1515, \\ Lomé, Togo. \\ ${ }^{2}$ Laboratoire de Chimie des Eaux, Faculté des Sciences, Université de Lomé, BP 1515, Lomé, Togo. \\ *Auteur correspondant, E-mail: kmadjouma@gmail.com ; BP. 20244, Lomé, Togo ; Tél. : +228 90161773.
}

\section{RESUME}

Cette étude vise à faire le point sur la diversité des espèces cultivées en agriculture maraîchère, leur distribution suivant les organes produits, la disponibilité des légumes ainsi que les contraintes qui entravent cette agriculture. Elle se fonde sur des enquêtes menées sous forme de "focus groups" auprès des maraîchers du pays. Les données recueillies et traitées ont révélé 43 espèces maraîchères qui se répartissent en 30 genres et 17 familles. Les légumes feuilles sont les plus fréquents quoique l'on puisse constater une prédominance de production de la carotte (Daucus carotta L.). Bien que Brassica oleracea var. capitata L. et Lactuca sativa L. soient cultivées dans tout le pays, elles semblent néanmoins être spécifiques des régions sèches de la Kara et des Savanes qui alimentent la capitale Lomé, en saison sèche (novembre à mars). Cette agriculture est soumise à des contraintes mais, celle qui semble avoir le plus d'impact est liée au foncier. En agissant sur celui-ci, on peut faire évoluer l'agriculture maraîchère au Togo vers des futurs prometteurs.

(C) 2014 International Formulae Group. All rights reserved.

Mots clés : Diversité, espèces cultivées, contraintes, agriculture maraîchère, Togo.

\section{INTRODUCTION}

Pour permettre aux villes africaines d'assurer une sécurité alimentaire à leurs populations, le développement de l'agriculture maraîchère demeure une solution de valeur. En Afrique, cette forme d'agriculture emploie une part importante des citadins (Delamarche, 2007). Dongmo et al. (2005) soutiennent que cette forme d'agriculture offre la possibilité d'un approvisionnement en produits frais. Une étude du Programme des Nations Unies pour le Développement (PNUD) a révélé que cette activité se pratique dans plus de 90 villes de
31 pays d'Asie du Sud-Est, du Moyen-Orient, d'Europe, d'Afrique subsaharienne, d'Amérique du Sud, du Centre, du Nord et des Antilles (Koc et al., 2006). Elle contribue à plus de $33 \%$ de la production agricole mondiale et emploie 800 millions de personnes.

Aujourd'hui, l'agriculture marâichère fait partie de l'environnement des villes africaines (Akinbamijo et al., 2002). La ville facilite l'écoulement de la production; cette agriculture a de faibles exigences en capital et en expertise ; les légumes s'adaptent mieux à 
l'alimentation urbaine; le cycle court des cultures légumières est un autre atout (Mougeot et Moustier, 2004).

En Afrique de l'Ouest, depuis son introduction dès la colonisation, l'agriculture maraîchère a pris un essor particulier avec le développement des villes et la demande croissante en produits maraîchers frais (Koné et al., 2000). Ainsi, le Togo a-t-il connu ces dernières années un développement du maraîchage urbain et périurbain. Les marchés de Lomé et des autres villes du pays sont approvisionnés en légumes frais issus de cette agriculture. Cette agriculture a progressé pour satisfaire une demande urbaine de plus en plus croissante ainsi que son importance dans l'économie nationale. Malheureusement, face à diverses contraintes, cette forme d'agriculture ne connaît pas le développement attendu.

La présente étude dont l'objectif est de contribuer à une meilleure connaissance de l'agriculture maraîchère au Togo afin de favoriser sa promotion et son développement, est entreprise pour :

- Déterminer la diversité des espèces cultivées dans les périmètres maraîchers et sa disponibilité ;

- Identifier les contraintes majeures à la production maraîchère.

\section{MATERIEL ET METHODES}

L'étude a couvert les cinq (5) régions administratives (Maritime, des Plateaux, Centrale, de la Kara et des Savanes) du Togo. Elle a été précédée d'une phase prospective exploratoire pour dresser une liste des zones de production maraîchère du pays à partir des données bibliographiques. En dehors des zones périurbaines, certains sites ruraux ont été retenus du fait de l'importance de leur production maraîchère (DESA, 1994). Ainsi, 27 sites de production maraîchère ont été retenus sur la base des critères suivant:

- l'accès à l'eau : les sites ont été retenus de manière à inclure différentes sources d'eau comme les rivières, les barrages, les puits, les marres et les forages;

- l'importance du site : ce critère tient compte du nombre de producteurs exerçant sur le site ou son importance en termes de superficie;

- les types de production maraîchère (la saisonnalité ou la permanence des activités maraîchères par site).

Sur chaque site, deux à trois périmètres maraîchers ont été prospectés et les maraîchers présents enquêtés. Les coordonnées géographiques de ces différents points d'observation ont été enregistrées au GPS.

La collecte des données est basée sur des enquêtes individuelles (Sinarinzi et Nisabw, 1999), complétées par des discussions de groupe ("focus groups"). Cette approche permet de mieux appréhender les connaissances, les attitudes, les pratiques et perceptions des groupes ciblés par rapport aux questions posées (Dawson et al., 1995).

Le questionnaire adressé au chef de l'exploitation ou à son représentant prend en compte les caractéristiques telles que:

- les caractéristiques socio-économiques des producteurs (sexe, âge, niveau d'instruction, type de formation, nombre d'années d'expérience, nombre d'actifs agricoles);

- les caractéristiques des différentes exploitations (la diversité de légumes cultivés, la taille de l'exploitation, les techniques culturales, les calendriers culturaux, quantité produite, le mode d'acquisition de la parcelle, la méthode d'irrigation); etc.

Les données d'enquêtes ont été complétées par des inventaires floristiques itinérants qui ont permis de dresser pour chaque enquêté, la liste des espèces cultivées, l'organe récolté, etc.

Une analyse botanique des espèces ainsi recensées a été faite afin de déterminer la diversité des espèces et variétés de plantes cultivées. La distribution des espèces suivant les organes consommés a été réalisée. Les 
espèces recensées ont été déterminées suivant la méthode de Dupriez et de Leerner (1987). La nomenclature suivie est celle de Hutchinson et Dalziel (1954-1972). Elle est ensuite corrigée avec la classification de l'Angiosperm Phylogeny Group III (2009).

Les données collectées ont été saisies dans un tableur Microsoft Excel ${ }^{\circledR}$, traitées et analysées par des statistiques descriptives afin de déterminer des moyennes et des fréquences. Des moyennes et des pourcentages ont été calculés et utilisés pour la construction d'histogrammes de distribution pour chacun des facteurs de production analysés, entre autres : le mode d'accès à la terre et les sources d'approvisionnement en eau.

La variabilité saisonnière de la production maraîchère, l'impact et les contraintes à la production maraîchère et à la commercialisation des légumes et les difficultés y afférentes ont été analysés.

Ces variables ont été analysées aussi bien au niveau national qu'au niveau des différentes régions administratives prospectées.

\section{RESULTATS}

Diversité des espèces cultivées

\section{Diversité et prédominance des produits maraîchers}

Cette étude a permis de recenser 43 espèces légumières reparties en 30 genres et 17 familles (Tableau 1). Le nombre d'espèces cultivées par maraîcher varie de 1 à 13 avec une moyenne de production de 5 espèces par champ. Les familles les plus représentées sont les Solanaceae (10 espèces), les Alliaceae (4), les Amaranthaceae, les Asteraceae, les Cucurbitaceae, les Lamiaceae et les Poaceae (3 espèces chacune). Les Apiaceae, les Brassicaceae, les Fabaceae et les Malvaceae sont représentées par deux (2) espèces chacune. Toutes les autres familles sont représentées par une seule espèce. Comme on peut le remarquer, la diversité spécifique est faible dans ces écosystèmes cultivés.
La distribution des fréquences spécifiques en fonction des maraîchers (Figure 1) montre qu'un petit nombre d'espèces est cultivé par la majorité des maraîchers alors que beaucoup d'espèces ont des fréquences faibles traduisant ainsi leur production par un petit nombre de maraîchers. Les espèces dont la fréquence relative est supérieure à $20 \%$ sont: Daucus carota (49\%), Solanum macrocarpum (46\%), Lactuca sativa (39\%), Lycopersicum esculentum (37\%), Corchorus olitorius (36\%) et Brassica oleracea (33\%).

\section{Distribution des espèces suivant les organes produits}

En fonction des organes consommés (Figure 2), la distribution des espèces montre que $51 \%$ des espèces sont cultivées pour leurs feuilles et $28 \%$ pour leurs fruits. La production maraîchère est orientée vers la production de légumes feuilles, de fruits et de racines pour respectivement $83 \%, 79 \%$ et $52 \%$.

Parmi les légumes feuilles, prédominent Solanum macrocarpum (46\%), Lactuca sativa (39\%), Corchorus olitorius (36\%) et Hibiscus sabdariffa (10\%). Les légumes fruits dominants sont Lycopersicum esculentum (37\%), Capsicum annuum (26\%), Capsicum annuum var. annuит (25\%), Cucumis sativus (19\%) et Abelmoschus esculentus (18\%). Les légumes racines sont représentés par Daucus carota (48\%) et Beta vulgaris (27\%) tandis que les légumes bulbes sont représentés par Allium cepa (22\%).

\section{Disponibilité des produits marâ̂chers}

De manière globale, la production maraîchère dure toute l'année pour la plupart des légumes, avec certes, une intensification observée de novembre à février (Tableau 2). Cette intensification de la production en cette période pourrait s'expliquer par le fait que ce moment de l'année coïncide avec :

- la période des fêtes de fin d'année et sa forte demande en produits maraîchers ;

- la saison morte des cultures vivrières

et l'arrivée des maraîchers saisonniers 
viennent "gonfler" les rangs des maraîchers habituels ;

- la période d'étiage des cours d'eau permettant une occupation des berges et des terrains inondés en période pluvieuse.

Se fondant sur les espèces à fréquence supérieure à $20 \% \quad$ (D. carota, $S$. macrocarpum, L. sativa, L. esculentum et $C$. olitorius), il a été possible d'établir la disponibilité de ces ressources suivant chaque région administrative.

Les régions sous climat plus ou moins sec tels que celles des Savanes, de la Kara et Centrale ont un climat de type soudanoguinéen avec des nuits assez fraîches, caractérisée par une saison pluvieuse et une saison sèche marquée par l'harmattan. Ces régions semblent se spécialiser pour certaines cultures telles que $S$. macrocarpum, $B$. oleracea var. capitata, $L$. sativa et $C$. olitorius. Il est à noter que la production de cette dernière espèce est faible à moyenne et semble être la seule espèce majeure de la région Centrale.

La région Maritime sous climat subéquatorial de transition (Papadakis, 1966 ; Trochain, 1980) est caractérisée par deux saisons pluvieuses et deux saisons sèches avec une faible pluviosité dépassant rarement 900 $\mathrm{mm} / \mathrm{an}$ et une humidité atmosphérique toujours supérieure à $70 \%$. Cette région est celle de la production de $S$. macrocarpum, $D$. carota et de $B$. oleracea var. capitata. La production de $B$. oleracea var. capitata dans cette région est en deçà de celle des trois autres régions citées plus haut.

La région des Plateaux renferme la zone IV qui reste la zone la plus arrosée de tout le pays. La pluviosité est rarement inférieure à 1200 mm/an et l'humidité atmosphérique, très élevée, dépasse toujours $70 \%$ sur au moins 8 mois. Elle produit toute l'année B. oleracea var. capitata. Quant à S. macrocarpum, elle connaît deux pics de forte production sur l'année.

Outre l'importance des facteurs climatiques dans la production maraîchère, il est à noter un autre facteur lié à la demande. En effet, la production de D. carota dans la région Maritime semble liée à la demande de ce produit par les consommateurs de Lomé, la plus grande agglomération du pays (DGSCN, 2011). D. carota est une production maraîchère très commune à Lomé et sa préfecture. Elle est souvent consommée crue et intervient également dans divers mets culinaires.

\section{Contraintes à la production maraîchère}

Les contraintes récurrentes dans les différentes régions économiques sont les attaques parasitaires et des ravageurs, les maladies, l'appauvrissement des sols, la divagation des animaux, le manque de main d'œuvre, l'approvisionnement en eau, les problèmes foncier et financier (Figure 3 ).

Attaques parasitaires et des ravageurs

La forte pression parasitaire signalée par plus de $90 \%$ des maraîchers entrave la production maraîchère. Plusieurs types de parasites et de ravageurs ont été cités par les maraîchers (Figure 4). Le nombre moyen de citation de ravageurs par maraîcher est de 2,14. Les plus fréquemment mentionnés sont : - les insectes représentant $74 \%$ de l'ensemble des ravageurs, ils causent le plus de dommages aux légumes et sont représentés par les chenilles (déprédatrices de feuilles et des fruits, les criquets (feuilles) et bien d'autres insectes tels que les mouches blanches, les fourmis, les papillons, les termites, les pucerons, les courtilières, les cigales, les punaises, les coccinelles et les thrips (qui s'attaquent aux feuilles) ;

- Les nématodes (17\%) sont présents dans le sol et induisent la formation de nombreux petits nœuds parasitaires sur les racines ;

- Les autres ravageurs regroupent les mammifères (rongeurs, chèvres, moutons, porcs, bœufs, singes), mollusques, bactéries, arachnides, champignons et oiseaux qui causent d'importants dégâts aux planches et aux cultures. 
Cette pression parasitaire limite le nombre de récoltes et déprécie la qualité des légumes, compromettant ainsi les rendements et les recettes.

\section{Maladies}

Elles constituent le second fléau qui handicape les efforts des maraîchers dont plus de 50\% les mentionnent (Tableau 3). Parmi les maladies fréquemment évoquées, les plus importantes sont : le flétrissement des feuilles, la rouille et la galle.

\section{Appauvrissement des sols}

La pauvreté des sols a été évoquée par l'ensemble des maraîchers et ce quel que soit le site de production. Dans la région forestière des Plateaux, les sols sont plus riches et permettent une agriculture maraîchère itinérante. L'appauvrissement des sols serait lié à la monoculture intensive caractéristique de l'agriculture maraîchère. De fait, presque tous les maraîchers utilisent divers types d'engrais.

\section{Divagation des animaux}

Les maraîchers doivent également conjuguer avec les perturbations causées par les animaux domestiques en divagation. Cette contrainte très variable est une préoccupation majeure pour les maraîchers des régions de la Kara $(79 \%)$, des Savanes $(77 \%)$ et Centrale (67\%) ; régions dans les quelles la divagation animale est préoccupante surtout en saison sèche.

\section{Manque de main d'œuvre}

Les résultats de nos enquêtes ont montré que la main-d'œuvre, en tant que facteur de production, peut être un facteur limitant dans un contexte d'accroissement des surfaces cultivables. Ce facteur quoiqu'ayant une incidence faible dans la région Maritime, devient remarquable dans les quatre autres régions agro-administratives du pays.

\section{Approvisionnement en eau}

À l'exception de la région Maritime où la nappe phréatique est à 2-5 mètres de profondeur, toutes les exploitations maraîchères sont situées le long des cours et plans d'eau saisonniers (rivières et barrages).
De ce fait, $53 \%$ des maraîchers confrontés au manque d'eau abandonnent temporairement l'activité en saison sèche (pouvant durer quatre (4) mois). Dans le cas contraire, certains $(10 \%)$ creusent des puits dans les lits desséchés. D’autres louent des motopompes (12\% des maraîchers dans la région Maritime) dont le coût est souvent difficile à supporter.

\section{Problème foncier}

L'accès à la terre est une contrainte majeure à l'essor de l'agriculture maraîchère au Togo qui justifie la taille globalement petite des surfaces emblavées. L'insécurité foncière rend les exploitations précaires et ne permet pas d'optimiser les rendements par des investissements durables (forage, utilisation de motopompes, etc.). Elle se traduit par de fréquents déguerpissements qui influencent la durée d'occupation agricole des parcelles de même que leur mobilité et leur instabilité. Cette difficulté est particulièrement très récurrente sur le littoral dans la région Maritime.

\section{Problème financier}

Le handicap financier, justifient les problèmes d'approvisionnement en intrants, demeure une préoccupation majeure pour les maraîchers. Les engrais NPK et l'urée couramment utilisés sont à coût prohibitif, rendant difficile l'accès à ces produits prioritaires pour des maraîchers, souvent confrontés à des ruptures de stock. Par ailleurs, l'engrais approprié aux cultures maraîchères (NPK 10 20) est introuvable sur le marché togolais. Ce qui oblige des maraîchers à se tourner vers des approvisionnements étrangers (Ghana, Burkina Faso et Bénin) coûteux. Il en est de même des semences et des produits phytosanitaires rarement disponibles, dont les prix restent hors de portée de la majorité des producteurs. Les semences utilisées ne garantissent pas une production de bonne qualité. Quant aux pesticides utilisés, ils ne sont pas homologués et peuvent avoir un impact négatif sur la santé des consommateurs et l'environnement. 
M. KANDA et al. / Int. J. Biol. Chem. Sci. 8(1): 115-127, 2014

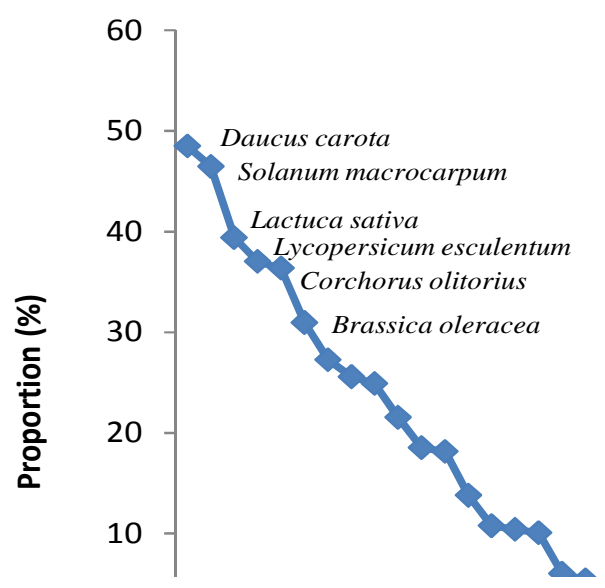

Figure 1: Diagramme rang fréquence des espèces maraîchères.

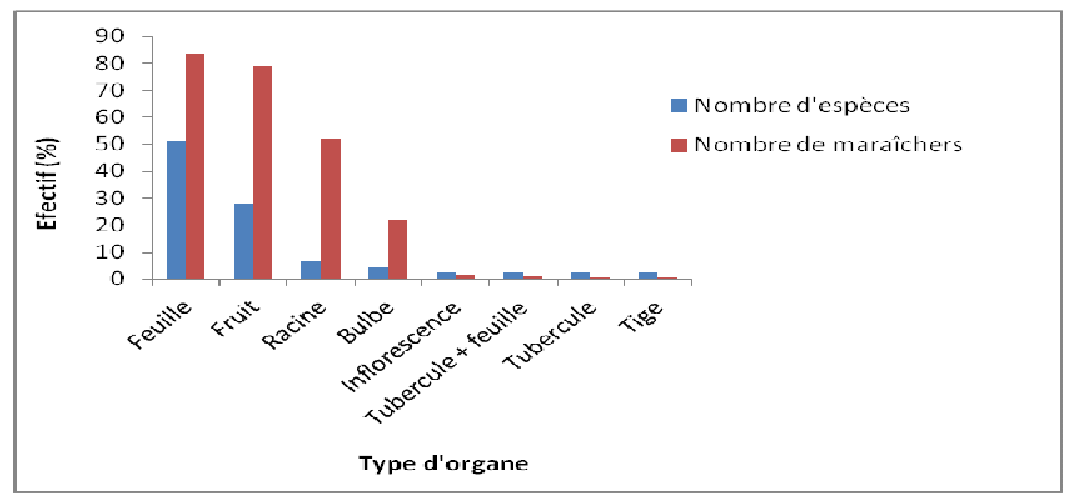

Figure 2: Répartition des espèces en fonction des organes consommés.

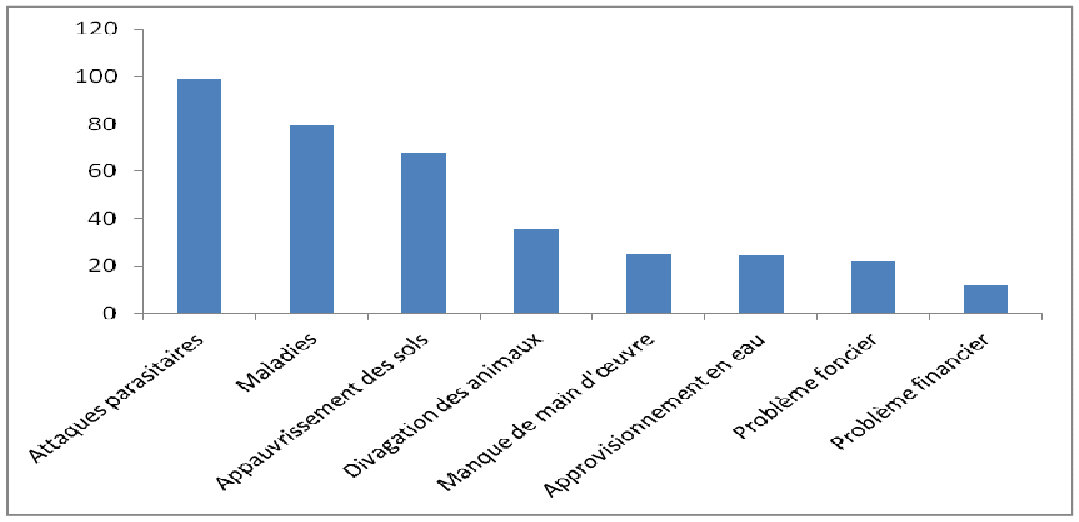

Figure 3: Principales contraintes à la production maraîchère au Togo. 


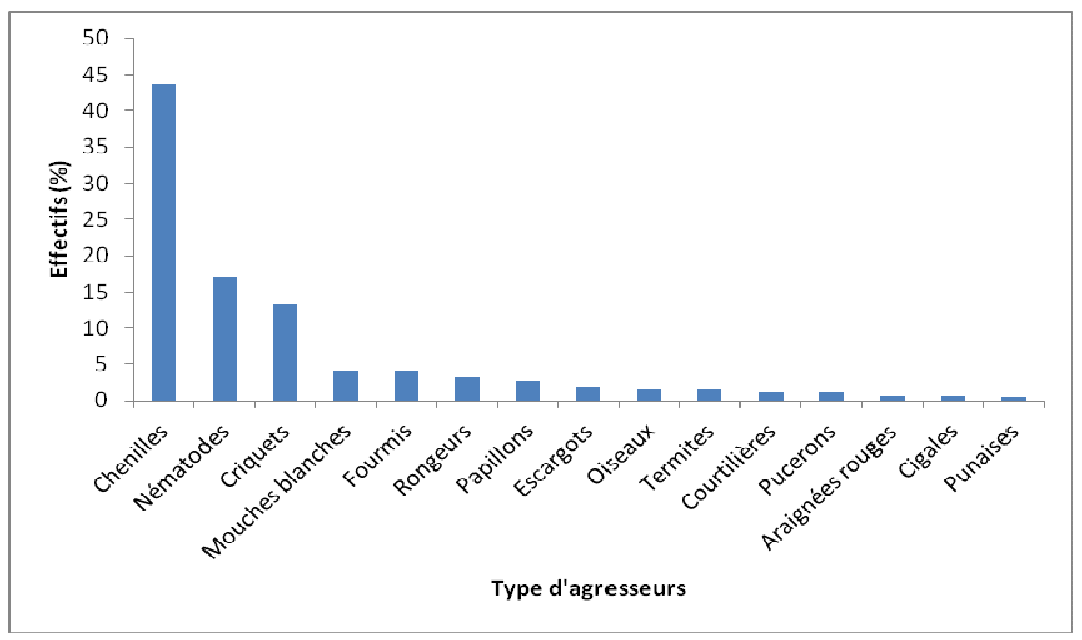

Figure 4: Principaux parasites et ravageurs en agriculture maraîchère au Togo.

Tableau 1: Diversité des espèces maraîchères cultivées.

\begin{tabular}{lcc}
\hline Familles & Nombre d'espèces & Nombre de genres \\
\hline Solanaceae & 10 & 4 \\
Alliaceae & 4 & 1 \\
Amaranthaceae & 3 & 2 \\
Asteraceae & 3 & 3 \\
Cucurbitaceae & 3 & 2 \\
Lamiaceae & 3 & 2 \\
Poaceae & 3 & 3 \\
Apiaceae & 2 & 2 \\
Fabaceae & 2 & 2 \\
Malvaceae & 2 & 2 \\
Brassicaceae & 2 & 1 \\
Capparaceae & 1 & 1 \\
Chenopodiaceae & 1 & 1 \\
Convolvulaceae & 1 & 1 \\
Pedaliaceae & 1 & 1 \\
Portucalaceae & 1 & 1 \\
Tiliaceae & 1 & 1 \\
\hline Total & 43 & 30 \\
\hline
\end{tabular}


Tableau 2: Période de production maraîchère au Togo.

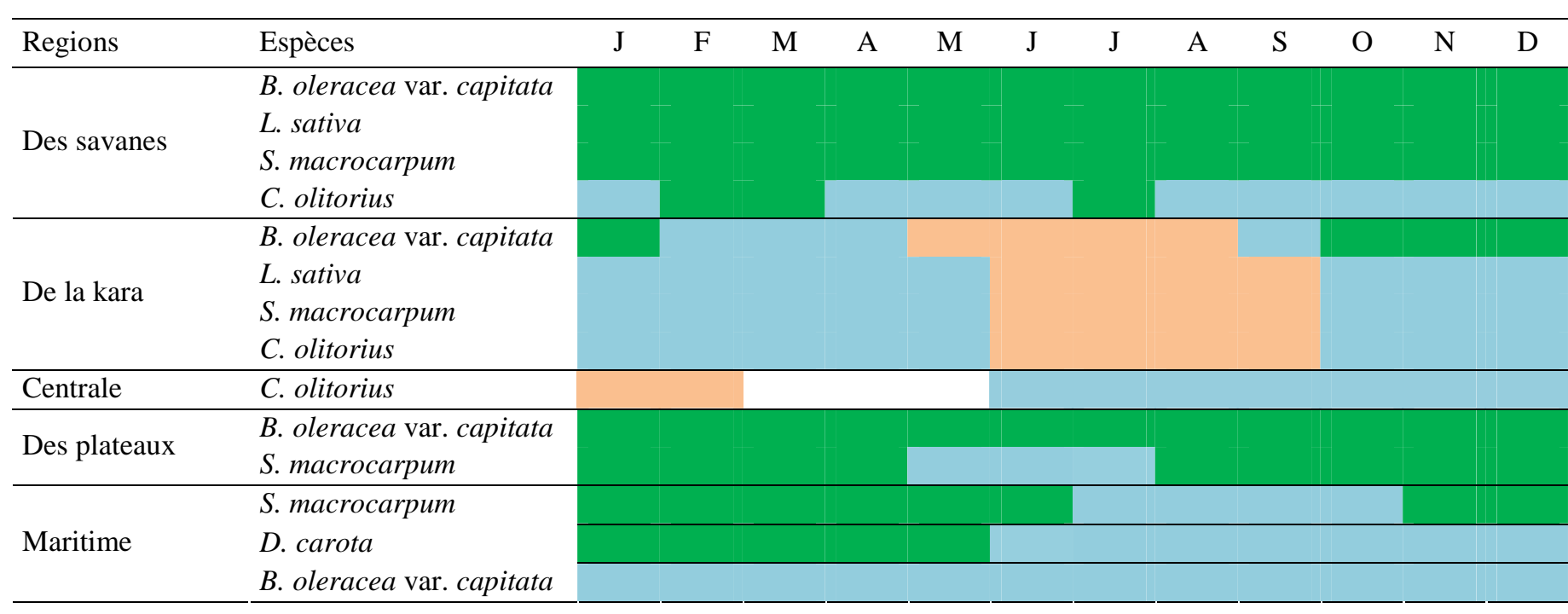

Forte production (par plus de 50\% des maraîchers)

Production moyenne (par 25\% à 50\% des maraîchers)

Production faible (par moins de 25\% des maraîchers)

Production nulle 
Tableau 3: Maladies fréquemment signalées.

\begin{tabular}{lc}
\hline Maladies & Fréquence relative \\
\hline Flétrissement des feuilles & 34,65 \\
Rouille & 33,07 \\
Galle & 11,42 \\
Pourritures & 8,27 \\
Flétrissement des pieds & 3,15 \\
Jaunissement des feuilles & 3,15 \\
Chute précoce de fruit & 2,36 \\
Maladie rouge & 1,18 \\
Trace & 1,18 \\
Fonte & 0,79 \\
Charbon & 0,39 \\
Tache noire & 0,39 \\
\hline
\end{tabular}

\section{DISCUSSION}

\section{Diversité des espèces cultivées}

La présente étude a montré que l'agriculture maraîchère au Togo est diversifiée (43 espèces légumières reparties en 30 genres et 17 familles). Ces résultats se rapprochent de ceux de Kanda (2009) qui, travaillant uniquement sur l'agriculture maraîchère urbaine à Lomé, a recensé respectivement 32 espèces légumières se répartissant en 23 genres et 14 familles. Afanou (2010) a par contre, recensé 23 espèces réparties en 17 genres et 13 familles. Cette différence peut être liée aux périodes au cours desquelles ces études ont été menées, mais également à la taille de l'échantillon.

Wéthé et al. (2001) mentionnent aussi cette diversité des légumes au Cameroun et au Burkina-Faso.

La diversité des espèces cultivées est liée à la croissance de la demande mais également au brassage des différentes communautés culturelles urbaines. Cette relation a déjà été mise en évidence au Togo par Tallaki (2005), au Sénégal par Diao (2004) et au Cameroun par Dongmo et al. (2005). Temple et Moustier (2004) soulignent à cet effet que les nouveaux modes de vie et les brassages culturels conduisent les populations urbaines à diversifier leur consommation alimentaire. Selon Temple et Moustier (2004), le mode de vie urbain s'accompagne très souvent d'une augmentation de la consommation en légumes.

La diversité des espèces légumières s'explique également par la diversité des organes recherchés. Les espèces cultivées pour leurs feuilles sont les mieux représentées par rapport à celles cultivées pour leur fruit, leur racine, leur bulbe ou leur tubercule. Ces résultats obtenus se rapprochent de ceux de $\mathrm{Ba}$ (2007) qui a obtenu 32 espèces maraîchères dont 11 légumes feuilles, 9 légumes fruits, 4 légumes bulbes, 4 légumes tubercules et 1 légume gousse. Les résultats présents montrent également la part importante des maraîchers qui cultivent les légumes feuilles (plus de $80 \%$ des maraîchers). Il en est de même à Dakar $(\mathrm{Ba}, 2007)$ et à Kinshasa (Muzingu, 2007). En effet, sur la base de l'occupation des sols de chaque type de légumes, Muzingu (2007) a montré que la production légumière à Kinshasa s'oriente essentiellement vers la culture des légumes feuilles $(87 \%)$. Cette forte représentativité des légumes feuille peut s'expliquer par la prédominance de sauces à base de feuilles dans les habitudes culinaires du Togo en particulier, et de l'Afrique de l'Ouest en 
général (Gockowski et al., 2003). En effet, les légumes sont nécessaires à l'équilibre du régime alimentaire. Ils sont riches en éléments nutritifs et contiennent des carotènes, la vitamine B (riboflavine et thiamine), la vitamine $\mathrm{C}$, des minéraux et des protéines (Amouzou et al., 2006) et conviennent aux enfants et aux personnes de santé fragile (Moustier et al. 2004). Ils contiennent aussi des éléments minéraux tels que le fer, le calcium et le phosphore (Mougeot, 2000). Les jardins maraîchers permettent ainsi aux ménages défavorisés de s'alimenter en légumes frais et d'améliorer la valeur des repas en protéines et en vitamines, tout en générant des revenus substantiels (Mougeot, 2000).

\section{Contraintes à la production maraîchère}

De nombreuses contraintes freinent le développement de l'agriculture maraîchère au Togo. Ces contraintes sont également constatées dans des études effectuées sur l'agriculture maraîchère en Afrique subsaharienne. De toutes les contraintes citées, il apparait que les attaques parasitaires, les difficultés d'accès au foncier, à l'eau et aux intrants sont les principales. Au Togo, Mondedji (2010) a spécifié que les ravageurs et les maladies des cultures constituent les contraintes majeures de l'agriculture maraîchère. Au Bénin, Tchuinte (2005) a montré que l'absence d'eau en saison sèche et la forte pression parasitaire entravent la production marâichère.

$\mathrm{La}$ pression parasitaire serait probablement liée aux pratiques culturales (précédents culturaux, absence de rotations, associations culturales liées à l'émiettement des surfaces cultivées, etc.). Plusieurs études ont signalé que la pression parasitaire est une contrainte importante pour l'agriculture maraîchère, particulièrement pendant la saison des pluies. Les maladies prédominent pendant la saison des pluies, alors qu'en saison sèche, les attaques par les insectes phytophages deviennent préoccupantes. L'intensité des dégâts occasionnés dépend largement de la capacité des maraîchers à maîtriser les techniques de lutte. Leur connaissance des ravageurs et des maladies mais surtout des méthodes de lutte est généralement absente ou limitée. De plus, les difficultés liées à l'acquisition d'intrants constituent un frein à une lutte efficace contre ces ravageurs (Huat, 2008). Au Bénin, Singbo et al. (2004) ont également mentionné que l'intensité de ces contraintes traduit le manque de moyens financiers, l'indisponibilité et le coût élevé des intrants.

En dehors des pressions parasitaires, la contrainte foncière est durement vécue par les maraîchers togolais. En effet, confrontés au problème d'accès au foncier, les maraîchers togolais exploitent des surfaces très variables de 0,9 are à 1200 ares. Selon Ba (2007), l'accès au foncier est la contrainte majeure dans beaucoup de pays africains et l'une des sources de différenciation des systèmes de production et des revenus. En Afrique subsaharienne, l'agriculture maraîchère se pratique sur des parcelles dont la superficie atteint rarement l'hectare (Mbaye et Moustier, 2000). Il est à relever que dans les villes, l'urbanisation accentue la contrainte foncière. L'insécurité foncière et la pénurie de terres consécutives à l'extension des zones bâties à vocation résidentielle, commerciale ou industrielle limitent le développement des activités maraîchères comme le soulignent Matthys et al. (2006).

\section{Conclusion}

La présente étude a permis de mettre en exergue quelques paramètres qui régissent la production maraîchère au Togo. La diversité des légumes recensés regroupe 43 espèces en 30 genres et 17 familles. Malgré une prédominance nette de la production de $D$. carotta, il est à observer que les légumes feuilles sont les plus cultivés car ils sont les plus demandés par le marché. 
Cette agriculture fait face à de nombreuses contraintes que sont les attaques parasitaires, les maladies, l'appauvrissement des sols, difficulté d'approvisionnement en eau, en intrants, les problèmes fonciers et les difficultés d'écoulement des produits. Pour que l'agriculture maraîchère puisse intégrer pleinement les programmes d'aménagement $\mathrm{du}$ territoire, il convient d'agir sur ces contraintes. Cette intégration est un impératif qui constitue un facteur fondamental du développement et de la satisfaction des besoins alimentaires des villes africaines. Pour ce faire, il faut, dans un système bien organisé:

- $\quad$ sécuriser l'accès au foncier et à l'eau ;

- prendre en compte la lutte contre les ravageurs ;

- mettre l'accent sur l'assurance-qualité de la production en agissant sur les bonnes pratiques permettant de réduire le recours aux produits phytosanitaires non autorisés.

\section{REFERENCES}

Afanou EE. 2010. La production maraîchère dans la préfecture des lacs et ses environs: implications socio-économiques et environnementales. Mém. Ing. Travaux, Ecole Supérieure des Techniques Biologiques et Alimentaires, Univ. Lomé (Togo), p. 39.

Akinbamijo OO, Fall ST, Smith OB. 2002. Advances in Crop-livestock Integration in West African Cities. ITC/ISRA/CRDI: Dakar.

Amouzou K, Adaké B, Batawila K, Wala K, Akpavi S, Kanda M, Odah K, KossiTitrikou K, Butaré I, Bouchet P, Akpagana K. 2006. Etudes biochimiques et évaluation des valeurs nutritionnelles de quelques espèces alimentaires mineures du Togo. Acta Bot. Gallica, 153(2): 147-152.

$\mathrm{Ba}$ A. 2007. Les fonctions reconnues à l'agriculture intra et périurbaines (AIPU) dans le contexte dakarois; caractérisation, analyse et diagnostic de durabilité de cette agriculture en vue de son intégration dans le projet urbain de Dakar (Sénégal). Thèse Doct. Sc. Agron. Environnement, AgroParisTech/UCAD/ENSP-Versailles, p. 357.

Dawson S, Manderson L, Tallo VL. 1995. The Focus Group Manual. Document produit par l'OMS, TDR/ SER/MSR/92.1.

Delamarche M. 2007. Agriculture urbaine, les villes qui se ruralisent. Marchés Tropicaux et Méditerranéens : Stratégies et Investissement en Afrique, 3212 : 5-6.

DESA (Direction des Enquêtes et des Statistiques Agricoles). 1994. Secteur maraîcher : une alternative aux problèmes d'emploi et à la sécurité alimentaire. Rapport, DESA éd., Lomé.

Diao MB. 2004. Situation et contraintes des systèmes urbains et périurbains de production horticole et animale dans la région de Dakar. Cah. Agric., 13: 39-49.

DGSCN (Direction Générale de la Statistique et de la Comptabilité Nationale). 2011. Recensement général de la population. Rapport, DGSCN éd., Lomé.

Dongmo T, Gockowski J, Hernandez S, Awono LDK, Mbang à Moudon R. 2005. L'agriculture périurbaine à Yaoundé : ses rapports avec la réduction de la pauvreté, le développement économique, la conservation de la biodiversité et de l'environnement. Tropicultura, 23(3) : 130-135.

Dupriez H, de Leerner P. 1987. Jardins et Vergers d'Afrique. Terres et Vie/L'Harmattan/APICA/ENDA/CTA (ed) ; 280.

Gockowski J, Mbazo'o J, Mbah G, Moulende TF. 2003. African traditional leafy vegetables and urban and peri-urban poor. Food Policy, 28: 221-235.

Huat J. 2008. Diagnostic sur la variabilité des modes de conduite d'une culture et de leurs conséquences agronomiques dans 
une agriculture fortement soumise aux incertitudes : cas de la tomate de plein champ à Mayotte. Thèse doct. Agronomie, Institut des Sciences et Industries du Vivant et de l'Environnement, AgroParisTech, p. 189.

Hutchinson J, Dalziel JM. 1954-1972. Flora of West Tropical Africa ( $2^{\text {nd }}$ edn, 3 vols). Crown Agents for Oversea Governments and Administrations: London.

Koc M, Macrae R, Mougeot JAL, Welsh J. 2000. Armer les Villes contre la Faim: Systèmes Alimentaires Urbains Durables. CRDI ; 243.

Koné D, Cissé G, Seignez C, Holliger C. 2000. Le lagunage à macrophytes et l'irrigation en maraîchage: étude d'une station expérimentale à Ouagadougou. Info CREPA, 31: 4-10.

Matthys B, Adiko FA, Cissé G, Wyss K, Tschannen AB, Tanner M, Utzinger J. 2006. Le réseau social des maraîchers à Abidjan agit sur la perception des préoccupations et des risques sanitaires liés à l'eau. VertigO, $H S, 3(8): 11$.

Mbaye A, Moustier P. 2000. Market-oriented urban agricultural production in Dakar. In Growing Cities, Growing Food: Urban Agriculture on the Policy Agenda, a Reader on Urban Agriculture. Bakker N, Dubbeling M, Gündel S, Sabel-Koschella U, de Zeeuw H (eds). Feldafing, Des/Etc: Allemagne, 235-257.

Mondedji AD. 2010. Potentiel d'utilisation d'extraits de feuilles de neem (Azadirachta indica A. Juss.) et de papayer (Carica papaya L.) dans le contrôle des insectes ravageurs du chou (Brassica oleracea L.) en zones urbaines et périurbaines au sud du Togo. Thèse de doctorat de l'Université de Lomé, p. 194

Mougeot J-AL, Moustier P. 2004. Introduction. In Développement Durable de l'Agriculture Urbaine en Afrique Francophone. Enjeux, concepts et méthodes. Olanrewaju BS, Moustier P,
Mougeot AJ-L, Fall A (eds). CIRAD/CRDI: Montpelier, 11-21.

Mougeot LJA. 2000. Urban agriculture: definition, presence, potential and risks. In Growing Cities, Growing Good: Urban Agriculture on the Policy Agenda, a Reader on Urban Agriculture. Feldafing : Allemagne.

Mougeot LJA. 2000. Urban agriculture: definition, presence, potential and risks. In Growing cities, growing food: urban agriculture on the policy agenda, a reader on urban agriculture. Bakker $\mathrm{N}$, Dubbeling M, Gündel S, Sabel-Koschella U, de Zeeuw H, (eds). Feldafing, Allemagne, Des/Etc, 123-142.

Moustier P, Moumbélé M, Huat J. 2004. La gestion concertée et durable des filières maraîchères urbaines In Développement Durable de l'Agriculture Urbaine en Afrique Francophone : Enjeux, Concepts et Méthodes (vol. 3), Olanrewaju BS, Moustier P, Mougeot LJA, Fall A, (eds). CRDI/CIRAD ; 79-114.

Muzingu BN. 2007. Comportement organisationnel des sites de coopératives maraîchères de Kinshasa vis-à-vis des contraintes environnementales. In Les Performances des Organisations Africaines. Pratiques de Gestion en Contexte Incertain, coll. Conception et dynamique des organisations, Nizet $\mathrm{J}$, Pichault F (eds). L'Harmattan : Paris ; 89106.

Papadakis J. 1966. Enquête Agroécologique en Afrique Occidentale (Liberia, C.I., Ghana, Togo, Dahomey, Nigeria). FAO : Rome.

Sinarinzi F, Nisabw T. 1999. Étude sur la Problématique des Terres Laissées par les Réfugiés de 1972 dans les Communes Rumonge et Nyanza-lac. Bujumbura, Burundi.

Singbo A, Nouhoheflin T, Idrissou L. 2004. Etude des Perceptions sur les Ravageurs des Légumes dans les Zones Urbaines et 
Périurbaines du Sud Bénin. Rapport d'activités, IITA - INRAB - OBEPAB, Cotonou, Bénin.

Tallaki K. 2005. The pest control systems in the market gardens of Lomé, Togo. In The Social, Political and Environmental Dimensions of Urban Agriculture, Mougeot L (ed). Earthscan: London.

Tchuinte GMS. 2005. Etude socioéconomique des systèmes de production maraîchère en zones urbaines et périurbaine de la ville de Djougou (Département de la Donga). Mém. Ing. Agron., FSA, Univ. Abomey-Calavi, Cotonou, p. 141

Temple L, Moustier P. 2004. Les fonctions et contraintes de l'agriculture périurbaine de quelques villes africaines (Yaoundé,
Cotonou, Dakar). Cahiers Agricultures, 13: $15-22$.

Temple L, Moustier P. 2004. Les fonctions et contraintes de l'agriculture périurbaine de quelques villes africaines (Yaoundé, Cotonou, Dakar). Cahiers Agricultures, 13: $15-22$.

Trochain JL. 1980. Ecologie Végétale de la Zone Intertropicale Désertique. UPS : Toulouse, France.

Wéthé J, Kientga M, Koné D, Kuéla N. 2001. Profil du Recyclage des Eaux Usées dans l'Agriculture Urbaine à Ouagadougou. Projet de recherche/consultation pour le développement durable de l'agriculture urbaine en Afrique de l'Ouest, IAGU/EIER/RFAU/AOC (éd), Yaoundé. 